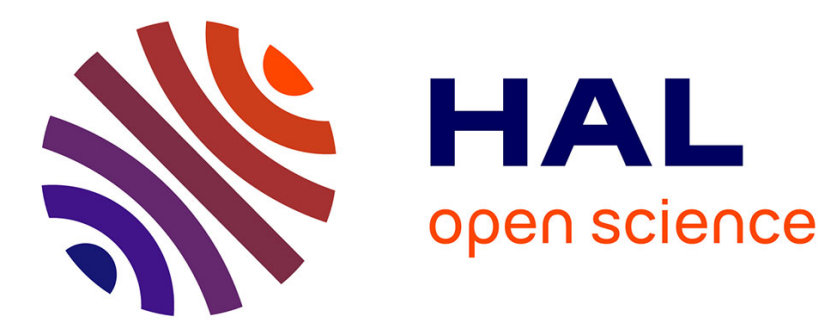

\title{
Performance of selection combining macro diversity with outage probability constraint in LPWAN
}

Qipeng Song, Xavier Lagrange, Loutfi Nuaymi

\section{To cite this version:}

Qipeng Song, Xavier Lagrange, Loutfi Nuaymi. Performance of selection combining macro diversity with outage probability constraint in LPWAN. ADHOC-NOW 2018: 17th International Conference on Ad Hoc Networks and Wireless, Sep 2018, Saint-Malo, France. 10.1007/978-3-030-00247-3_18 . hal-01897579

\section{HAL Id: hal-01897579 \\ https://imt-atlantique.hal.science/hal-01897579}

Submitted on 17 Oct 2018

HAL is a multi-disciplinary open access archive for the deposit and dissemination of scientific research documents, whether they are published or not. The documents may come from teaching and research institutions in France or abroad, or from public or private research centers.
L'archive ouverte pluridisciplinaire HAL, est destinée au dépôt et à la diffusion de documents scientifiques de niveau recherche, publiés ou non, émanant des établissements d'enseignement et de recherche français ou étrangers, des laboratoires publics ou privés. 


\title{
Performance of selection combining macro diversity with outage probability constraint in LPWAN
}

\author{
Qipeng Song ${ }^{1,2}$, Xavier Lagrange ${ }^{1,2}$, and Loutfi Nuaymi ${ }^{1,2}$ \\ 1 IMT Atlantique, Cesson-sevigne 35510, France \\ ${ }^{2}$ IRISA, Cesson-Sevigne 35576, France
}

\begin{abstract}
In low power wide area networks (LPWAN), a packet transmitted by a device can be received and decoded by all surrounding base stations (BS). If at least one of the base stations decodes the packet, the latter is delivered to the system. This scheme is called selection combining (SC) macro diversity and has been studied in the literature. However, the existing research only cares about the average packet-loss probability over the entire networks. In this paper, we consider as the main performance indicator the outage probability, which better takes into account the real availability of the service in any part of the area: the outage probability. Given an outage probability constrain, we study the minimum required BS spatial density of SC macro diversity based LPWAN. The numerical results show that if both target packet loss rate and outage probability are $10 \%$, the minimum BS spatial density required by SC macro diversity is at most half of that required by traditional BS attach method. The performance gain is more significant when the network load increases.
\end{abstract}

Keywords: macro diversity $\cdot$ selective combining $\cdot$ LPWAN.

\section{Introduction}

Low Power Wide Area Network (LPWAN) is regarded as a promising solution to handle future machine type communication (MTC) traffic [12]. There are several technologies, but most representative ones (Sigfox and LoRaWAN) are based on Aloha because of its simplicity [10].

In cellular networks, a device always attaches to the Base Station(BS) for which the received power averaged over all fading realizations is the strongest and then transmits packets to its attached BS. This scheme is referred to as Best BS attach mode. However, a device in LPWAN works in a different way: it sends a packet in broadcast mode without BS attach procedure and benefits from macro reception diversity, which is defined as the capacity of several BS to receive the same packet (see Fig. 1). Each BS autonomously and independently decodes the packets and then sends the decoded packets to the core network. That is to say, each BS is equipped with a packet decode function, and the backhaul link between $\mathrm{BS}$ and core network transmits only when a packet is received at the 
BS. The core network is in charge of duplicate received packets removal (e.g., by comparing the identity and message content conveyed in packets). A packet is successfully delivered if at least one BS decodes the packets. This scheme is referred to as selection-combination-based macro diversity, simply written as $\boldsymbol{S C}$ macro diversity. It is currently used by Sigfox and LoRaWAN [10].

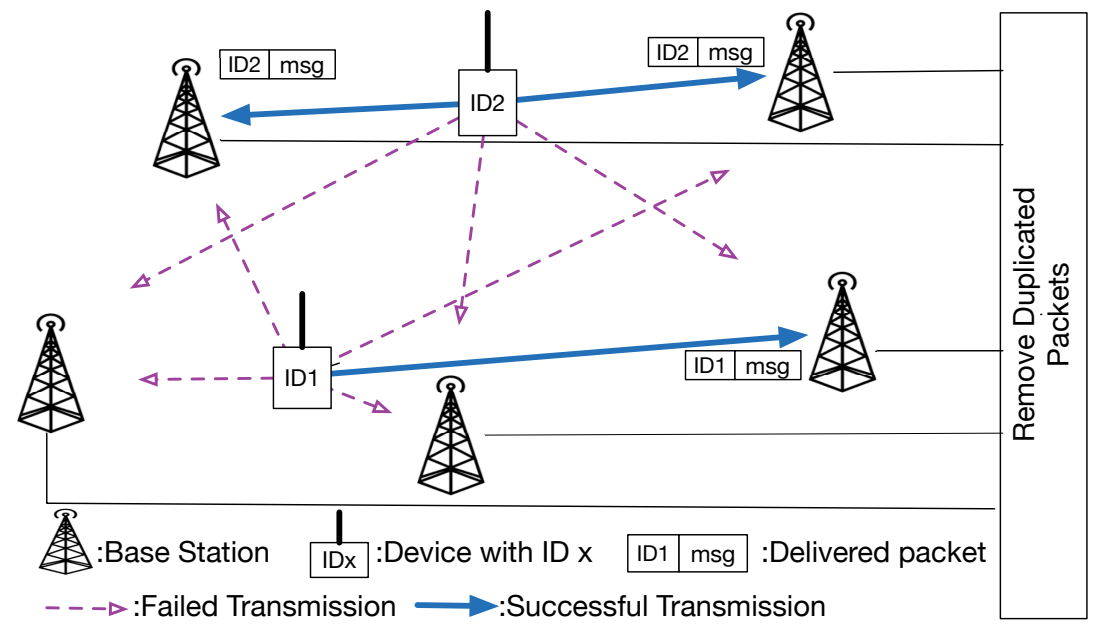

Fig. 1. Macro Reception Diversity Scheme Illustration

In the literature, the performance of ALOHA is usually evaluated with indicator (e.g. packet loss rate) that is averaged over the entire networks. This kind of indicator gives a global view about the quality of service in LPWAN networks but still have its limitations. For example, with a target network level packet loss rate such as $10 \%$, it is very possible that devices in the neighborhood of BS never fail while devices at the border of BS coverage area have packet loss rate higher than $10 \%$.

In this paper, we consider a performance indicator that better takes into account the real availability of the service in any part of the area: the outage probability. This indicator is defined as the probability that at any location a device has a link-level packet loss rate greater than a target value. For example, the statement that a LPWAN network has an outage probability of $10 \%$ with target packet loss rate $1 \%$, means that at any location, the probability that a device has a packet loss rate higher than $1 \%$ is $10 \%$. With outage probability, we can study the minimum BS spatial density if a LPWAN operator needs to guarantee a certain level of grade of service (i.e. outage probability). These studies can help LPWAN operators to deploy their network in an efficient way.

Combining techniques were studied to improve the performance of cellular networks in the 1990s. For example, the performance of SC macro diversity is studied for GSM networks with a remote antennas system by using hexagonal topology [7]. The packet loss rate and system capacity in ALOHA networks 
are usually analyzed with Stochastic geometry. The performance of SC macro diversity for BS with multiple receiver branches was studied in [9]. Stochastic geometry has served as a powerful tool to study the performance of LPWAN. In this field, Baccelli et al. [2] use Laplace transform for interference analysis for slotted ALOHA. Haenggi et al. [6] extensively study the outage probability in SINR-based capture model for slotted ALOHA. Błaszczyszyn et al. [3] open the door to study pure ALOHA with stochastic geometry. In our previous work [11], we neglected the background noise and obtained closed-form expression of average packet loss rate in SC macro diversity based Aloha LPWAN and showed the performance gain compared to best BS attach. However, to our best knowledge, $\mathrm{SC}$ macro reception diversity with outage probability for ALOHA systems has not been studied in literature.

The remaining of this paper is organized as follows: Sec. 2 introduces the system model in which we take into account the Rayleigh fading, shadowing, background noise and capture effect. Sec. 3 analyzes the successful packet transmission probability for a given pair of device and BS based on some stochastic geometry research results. These analyzes cover both pure and slotted ALOHA. Sec. 4 presents our analysis about the minimum required BS spatial density under outage probability constraint. Sec. 5 presents numerical results and discussions. Sec. 6 concludes this paper.

\section{System model}

For any random variable $(\mathrm{RV}) X$, let $f_{X}(x), F_{X}(x), \mathcal{L}_{X}[s], \phi_{X}(\omega)$ be its probability density function (PDF), cumulative distribution function (CDF), Laplace transform (LT) and characteristic function $(\mathrm{CF})$, respectively.

\subsection{Distribution of Nodes and Traffic Model}

We consider a large wireless network over a two-dimension infinite plane. The locations of terminals form a stationary Poisson point process (PPP) $\Phi_{m}=\left\{x_{i}\right\}$ on the plane $\mathbb{R}^{2}$, where $x_{i}$ refers to the coordinates of device with label $i$. The spatial density of $\Phi_{m}$ is $\lambda_{m}$. Similarly, the locations of base stations also form a stationary $\operatorname{PPP} \Phi_{b}=\left\{y_{j}\right\}$ with spatial density $\lambda_{b}$.

\subsection{Slotted ALOHA and Pure ALOHA}

In the system model, we consider pure ALOHA (P-ALOHA) and slotted ALOHA (S-ALOHA). For slotted ALOHA, the time domain is equally divided into slots with duration $T_{\text {slot }}$. In each slot, each device independently decides to transmit a packet with probability $p$. The propagation delay is assumed to be much smaller than $T_{\text {slot }}$. Hence, there is a global slot synchronization over the whole network. In pure ALOHA, devices send packets without synchronization, but we still use parameter $p$. The packet generation process can be seen as an internal slotted system in each device with $p$ the probability to transmit in a slot of duration 
$T_{\text {slot }}$. At a given time, the locations of terminals that are transmitting a packet form a thinned PPP with spatial density $p \lambda_{m}$. For slotted and pure ALOHA, we define the normalized load (per BS) as $L=p \lambda_{m} / \lambda_{b}$.

\subsection{Propagation model}

The propagation model is based on Okumura-Hata with both shadow and Rayleigh fading. We assume that MTC devices transmit with identical power level denoted by $P_{t}$. The received power $P_{r}$ of a packet at the BS is given by:

$$
P_{r}=P_{t} K r_{g}{ }^{-\gamma} H 10^{\sigma_{\mathrm{dB}} \chi / 10}=P_{t} K r_{g}{ }^{-\gamma} H \exp \left(\sigma_{\mathrm{dB}} \frac{\ln (10)}{10} \chi\right),
$$

where $K$ is a constant which depends on the antenna characteristics and the average channel attenuation, $r_{g}$ refers to the Euclidean distance between the transmitter and the receiver, $\gamma$ is the path-loss exponent, $H$ is an exponentially distributed $\mathrm{RV}$ with mean 1 , and $\chi$ is a standard normal variable. For the sake of simplification, we define $\sigma=\sigma_{\mathrm{dB}} \ln (10) / 10$. We assume that $H$ and $\chi$ are both constant during a packet transmission, and mutually independent for different links.

Capture effect is taken into account to determine whether a BS could decode a received packet. Thus, the transmission success probability $p_{s}$ for a transmitreceiver pair is defined as follows:

$$
p_{s}=\mathbb{P}\left\{\mathrm{SINR}=\frac{P_{r}}{I+\sigma_{n}^{2}} \geq \theta_{T}\right\}
$$

where $I$ refers to the suffered cumulative interference during packet transmission, $\sigma_{n}^{2}$ is the background noise power.

\subsection{Displacement Theorem}

To facilitate the analysis, we use the displacement theorem, which is formulated as a lemma in [4, lemma 1$]$.

Lemma 1. For a homogeneous PPP $\Phi \subset \mathbb{R}^{2}$ with spatial density $\lambda_{b}$, if each point $r_{g} \in \Phi$ is transformed to $r \in \mathbb{R}^{2}$ such that $r=X^{-\frac{1}{\gamma}} r_{g}$, where $\{X\}$ are i.i.d., such that $\mathbb{E}\left[X^{-\frac{2}{\gamma}}\right]<+\infty$, the new point process $\Phi^{\prime} \subset \mathbb{R}^{2}$ defined by the transformed points $r$ is also a homogeneous PPP with density $\lambda_{b}^{\prime}=\lambda_{b} \mathbb{E}\left[X^{\frac{2}{\gamma}}\right]$.

We thus rewrite (1) as follows:

$$
P_{r}=P_{t} K H r^{-\gamma} \text {, with } r=P_{t} K \exp (-\chi / \gamma) r_{g},
$$

which indicates that the performance analysis in the initial PPP with shadowing is equivalent to that in a transformed PPP of intensity $\lambda_{b}^{\prime}=\lambda_{b} \mathbb{E}\left[e^{\frac{2}{\gamma} \chi}\right]=\lambda_{b} e^{\frac{2 \sigma^{2}}{\gamma^{2}}}$ 
without shadowing but with the modified distance $r$. The PDF of $r$, proved in [1], is given as follows:

$$
f_{r}(r)=2 \pi \lambda_{b}^{\prime} \exp \left(-\lambda_{b}^{\prime} \pi r^{2}\right) r, r \in[0,+\infty],
$$

In the following, all distances are the modified ones in the transformed PPP.

\section{Link-level Transmission Success Probability}

We first study the transmission success probability $p_{s}(r)$ for a given uplink between a device with label $x_{0}$ and a device with label $y_{0}$. The distance between $x_{0}$ and $y_{0}$ is denoted by $r$. For the considered device $x_{0}$, all the other interfering devices constitute a PPP $\Phi_{m} \backslash\left\{x_{0}\right\}$. Combining (1) and (2), we have:

$$
p_{s}(r)=\mathbb{P}\left\{\frac{P_{t} K H r^{-\gamma}}{\sum_{x_{j} \in \Phi_{m} \backslash\left\{x_{0}\right\}} P_{t} K H_{x_{j}} r_{x_{j}}^{-\gamma}+\sigma_{n}^{2}} \geq \theta_{T}\right\},
$$

Using Slivnyak's theorem [14][Theorem 2.3.3], (5) can be further simplified:

$$
p_{s}(r)=\mathbb{P}\left\{\frac{P_{t} K H r^{-\gamma}}{\sum_{x_{j} \in \Phi_{m}} P_{t} K H_{x_{j}} r_{x_{j}}^{-\gamma}+\sigma_{n}^{2}} \geq \theta_{T}\right\} .
$$

Let $I=\sum_{x_{j} \in \Phi_{m}} P_{t} K H_{x_{j}} r_{x_{j}}^{-\gamma}$, which is the cumulative interference suffered by $x_{0}$. As shown in [6], $p_{s}(r)$ can be expressed in terms of Laplace transform of cumulative interference $\mathcal{L}_{I}(s)$ at point $\theta_{T} r^{\gamma}$ :

$$
\begin{aligned}
p_{s}(r) & =\mathbb{P}\left\{H \geq\left(I+\frac{\sigma_{n}^{2}}{P_{t} K}\right) \theta_{T} r^{\gamma}\right\} \\
& =\exp \left(-\frac{\sigma_{n}^{2} \theta_{T}}{P_{t} K} r^{\gamma}\right)\left[\int_{0}^{+\infty} \exp \left(-y \theta_{T} r^{\gamma}\right) f_{I}(y) d y\right] \\
& =\exp \left(-\frac{\sigma_{n}^{2} \theta_{T}}{P_{t} K} r^{\gamma}\right) \mathcal{L}_{I}\left\{\theta_{T} r^{\gamma}\right\}
\end{aligned}
$$

Now the problem is down to calculate the Laplace transform of cumulative interference suffered by the BS at the origin.

Interference $I$ in 2 is constant during a packet transmission for S-ALOHA, but may vary with respect to time for P-ALOHA because other MTCs start or stop their transmissions during the packet transmission. With advanced transmission techniques (e.g., interleaving, robust channel coding, etc.), $I$ is the average value within the considered packet transmission and the access is called Pure Aloha with average interference (Pa-ALOHA). For both access schemes, the Laplace transmission of $I$, proved in [3], is as follows:

$$
\mathcal{L}_{I}(s)=\exp \left\{-p \lambda_{m} \pi A \exp \left(2 \sigma^{2} / \gamma^{2}\right) s^{2 / \gamma}\right\},
$$


where $A$ is defined as follows:

$$
A=\left\{\begin{array}{cl}
\Gamma(1-2 / \gamma) \Gamma(1+2 / \gamma), & \text { for S-ALOHA } \\
\frac{2 \gamma}{\gamma+2} \Gamma(1-2 / \gamma) \Gamma(1+2 / \gamma), & \text { for Pa-ALOHA }
\end{array}\right.
$$

where $\Gamma(\cdot)$ is the gamma function.

For P-ALOHA without advanced transmission techniques, $I$ is the maximum interference value during the packet transmission and is called maximum interference (Pm-ALOHA). Its closed-form expression of LT does not exist [3]. In [11, Sec.III-B], we propose to approximate $I_{y_{j}}$ by un upper bound that is the sum of interference levels at the start and end time of the considered packet transmission. With our proposed proposed upper bound, (8) is extended to cover the case of Pm-ALOHA with $A=2 \Gamma(1-2 / \gamma) \Gamma(1+2 / \gamma)$.

By combining (7) and (8), we can generalize the link-level success transmission probability $p_{s}(r)$ for all ALOHA cases (slotted and pure ALOHA) :

$$
\begin{aligned}
p_{s}(r) & =\exp \left(-\frac{\sigma_{n}^{2} \theta_{T}}{P_{t} K} r^{\gamma}\right) \exp \left(-p \lambda_{m} \pi A \theta_{T}^{\frac{2}{\gamma}} e^{\frac{2 \sigma^{2}}{\gamma^{2}}} r^{2}\right) \\
& =\exp \left(-\eta_{T} r^{\gamma}\right) \exp \left(-\epsilon_{T} r^{2}\right)
\end{aligned}
$$

where $\sigma_{n}^{2}$ is the background noise power, $\theta_{T}$ is the capture ratio, $P_{t}$ refers to the device transmit power, $K$ is a positive constant related to propagation model

and constant $A$ is defined in (8). For the simplicity of notation, let $\eta_{T}=\frac{\sigma_{n}^{2} \theta_{T}}{P_{t} K}$, $\epsilon_{T}=p \lambda_{m} \pi A \theta_{T}^{\frac{2}{\gamma}} e^{\frac{2 \sigma^{2}}{\gamma^{2}}}$.

\section{Minimum required BS density}

The transmission success probability $p_{s}(r)$ paves the way to study the minimum required spatial density $\lambda_{b, \text { min }}$ for best BS attach and SC macro diversity.

\subsection{Best BS Attach}

Consider a device at the origin. If best BS attach method is applied, according to (9), the link level packet loss rate $p_{f, b}(r)$ is:

$$
p_{f, b}(r)=1-\exp \left(-\eta_{T} r^{\gamma}\right) \exp \left(-\epsilon_{T} r^{2}\right),
$$

where $\eta_{T}=\frac{\sigma_{n}^{2} \theta_{T}}{P_{t} K}, \epsilon_{T}=p \lambda_{m} \pi A \theta_{T}^{\frac{2}{\gamma}} e^{\frac{2 \sigma^{2}}{\gamma^{2}}}$.

Let $P_{f, \text { max }}$ be the target network loss rate and $P_{\text {outage }, b}$ be the outage probability (the subscript $b$ refers to the best BS attach method). Since the link level packet loss rate $p_{f, b}(r)$ is a function of $r$, thus, the proportion of devices that have higher packet loss rate than $P_{f, \max }$, namely the outage probability $P_{\text {outage }, b}$, 
can be expressed as follows:

$$
\begin{aligned}
P_{\text {outage }, b} & =\int_{0}^{+\infty} \mathbb{1}_{r}\left[p_{f, b}(r)>P_{f, \max }\right] f(r) d r=\int_{r_{c}}^{+\infty} f(r) d r \\
& =1-\int_{0}^{r_{c}} f(r) d r=\exp \left(-\lambda_{b} e^{\frac{2 \sigma^{2}}{\gamma^{2}}} \pi r_{c}{ }^{2}\right),
\end{aligned}
$$

where $\mathbb{1}_{r}[\cdot]$ is a indicator function about $r$ and its $\mathrm{PDF}$ is given in (4), $r_{c}$ is the critical distance that can be numerically calculated from equation $p_{f, b}=P_{f, \max }$. The critical distance $r_{c}$ is calculated by substituting $p_{f, b}\left(r_{c}\right)=P_{f, \max }$ into (10).

$$
1-\exp \left(-\eta_{T} r_{c}^{\gamma}\right) \exp \left(-\epsilon_{T} r_{c}^{2}\right)=P_{f, \max }
$$

Although there is no closed-form expression for critical distance $r_{c}$, the latter can be easily numerically calculated by leveraging the fact that $p_{f, b}(r)$ is a monoincreasing function with respect to $r$.

With the obtained $r_{c}$ and a given outage probability, the minimum BS spatial density $\lambda_{b, \text { min }}$ can be obtained by inversing (11):

$$
\lambda_{b, \text { min }}=-\ln \left(P_{\text {outage }, b}\right) \frac{1}{\pi \exp \left(2 \sigma^{2} / \gamma 2\right) r_{c}^{2}},
$$

where $\ln (\cdot)$ is the natural logarithm operator.

Without background noise In an interference-limited LPWAN system, the background can be reasonably neglected. Closed-form expression $r_{c}$ can be obtained from (12) by letting $\eta_{T}=0$ :

$$
r_{c}=\left[\ln \left(1-P_{f, \max }\right) /\left(-p \lambda_{m} \pi A \theta_{T}^{\frac{2}{\gamma}} e^{\frac{2 \sigma^{2}}{\gamma^{2}}}\right)\right]^{1 / 2}
$$

With substitution of (14) into (13), $\lambda_{b, \min }$ is as follows:

$$
\lambda_{b, \min }=p \lambda_{m} \frac{\ln \left(P_{\text {outage }, b}\right) A \theta_{T}^{2 / \gamma}}{\ln \left(1-P_{f, \max }\right)} .
$$

\subsection{SC Macro Reception Diversity}

Different from best BS attach method, whose link-level packet loss rate only depends on the distance to the nearest BS, the performance of SC macro reception diversity depends on a distance vector $r_{i}, i=0,1,2 \ldots$ Thus, we need to know the characteristics of such a vector. However, the distance to the nearest BS $r_{0}$ has the most impact to SC macro reception diversity. Thus, to reduce the complexity and keep the tractability, for a given device, its packet loss rate can be approximately expressed in terms of $r_{0}$ (instead of the distance vector to all surrounding $\left.\mathrm{BS} r_{i}, i=1,2 \ldots\right)$. 
Let $p_{f, s c}\left(r_{0}\right)$ be link-level packet loss rate with respect to $r_{0}$. Recall that in case of SC macro reception diversity,

$$
p_{f, s c}\left(r_{0}\right) \approx\left(1-p_{s}\left(r_{0}\right)\right) \mathbb{E}\left[\prod_{r_{i} \in \Phi_{b}}\left(1-p_{s}\left(r_{i}\right)\right)\right], \text { with } r_{i} \in\left[r_{0},+\infty\right],
$$

where $r_{i}$ refers to the distance between the device and BS with label $i$. Note that $r_{i}$ should be not less than $r_{0}$. The impact of BS other than the nearest one to the packet loss rate is thus reflected by term $\mathbb{E}\left[\prod_{r_{i} \in \Phi_{b}}\left(1-p_{s}\left(r_{i}\right)\right)\right]$.

Term $\mathbb{E}\left[\prod_{r_{i} \in \Phi_{b}}\left(1-p_{s}\left(r_{i}\right)\right)\right]$ in (16) is actually the Probability Generating FunctionaL (PGFL) of PPP $\Phi_{b}$ for BS, which states for some function $f(x)$ that:

$$
\mathbb{E}\left[\prod_{x \in \Phi} f(x)\right]=\exp \left(-\lambda\left(\int_{\mathbb{R}^{2}}(1-f(x)) d x\right)\right)
$$

Thus, it can be simplified as follows:

$$
\mathbb{E}\left[\prod_{r_{i} \in \Phi_{b}}\left(1-p_{s}\left(r_{i}\right)\right)\right]=\exp \left\{-\pi \lambda_{b} e^{\frac{2 \sigma^{2}}{\gamma^{2}}} \int_{r_{0}{ }^{2}}^{+\infty} \exp \left(-\eta_{T} r^{\frac{\gamma}{2}}-\epsilon_{T} r\right) d r\right\}
$$

With substitution of (17) into (16), we have:

$$
p_{f, s c}\left(r_{0}\right)=\left(1-e^{-\eta_{T} r_{0}^{\gamma}-\epsilon_{T} r_{0}^{2}}\right) \exp \left\{-\pi \lambda_{b} e^{\frac{2 \sigma^{2}}{\gamma^{2}}} \int_{r_{0}{ }^{2}}^{+\infty} \exp \left(-\eta_{T} r^{\frac{\gamma}{2}}-\epsilon_{T} r\right) d r\right\}
$$

By letting $p_{f, s c}\left(r_{0}\right)=P_{f, \max }$ and substituting (13) into (18), the critical distance $r_{c}$ can be numerically calculated due to the fact that $p_{f, s c}\left(r_{0}\right)$ is a monoincreasing function (even though its closed-form expression does not exist). Then we can use formula (13) to calculate the minimum BS spatial density, given an outage probability and target network packet loss rate.

\section{$5 \quad$ Numerical result}

The system settings to get the numerical results are resumed in Tab. 1. Some explanations are given as follows: to evaluate the impact of the background noise to the system performance, we need to determine the values of $P_{t}, K, \sigma_{n}^{2}$. For one LPWAN device running on ISM band (e.g. with $868 \mathrm{MHz}$ ), from a regulatory point of view, the effective radiated power (ERP) may not exceed $14 \mathrm{dBm}$ (or $25 \mathrm{~mW}$ ) in any direction [13], we thus assume that the transmit power $P_{t}$ is set as $14 \mathrm{dBm}$.

About noise power level, we use the same formula given in [5]: $\sigma_{n}^{2}=-174+$ $\mathrm{NF}+10 \log (B)$, where NF is the noise figure, usually set as $6 \mathrm{~dB}, B$ is the bandwidth occupied by the transmitted packet. The occupied transmission band is assumed to be $200 \mathrm{kHz}$ (e.g. LoRaWAN). Thus, $\sigma_{n}^{2}$ is $-115 \mathrm{dBm}$.

In terms of propagation path-loss model, Okumura-Hata model is applied. In urban area, the path-loss between one device with antenna height $h_{d}=1.5$ 
Table 1. Parameter values for numerical results

\begin{tabular}{lll}
\hline Parameter Name & \multicolumn{2}{l}{ Parameter Notation Value } \\
\hline Transmit power & $P_{t}$ & $14 \mathrm{dBm}$ \\
Noise figure & $\mathrm{NF}$ & $6 \mathrm{~dB}$ \\
Occupied bandwidth & $B$ & $200 \mathrm{kHz}$ \\
Target network packet loss rate & $P_{\mathrm{f}, \max }$ & $10 \%$ \\
Outage probability & $P_{\text {outage }}$ & $1 \%, 10 \%$ \\
Device height & $h_{d}$ & $1.5 \mathrm{~m}$ \\
BS antenna height & $h_{b s}$ & $50 \mathrm{~m}$ \\
Noise power & $\sigma_{n}^{2}$ & $-115 \mathrm{dBm}$ \\
\hline
\end{tabular}

meters and a BS with height $h_{b s}=50$ meters is [8]: Path_Loss $=-10 \log _{10}(K)+$ $\gamma 10 \log _{10}(r)=123.6+33.8 \log (r)$, where $\log _{10}(\cdot)$ is 10 -based logarithm operator, $r$ is in unit of kilometer. Thus, the constant $10 \log _{10}(K)$ is $-123.6 \mathrm{~dB}$.

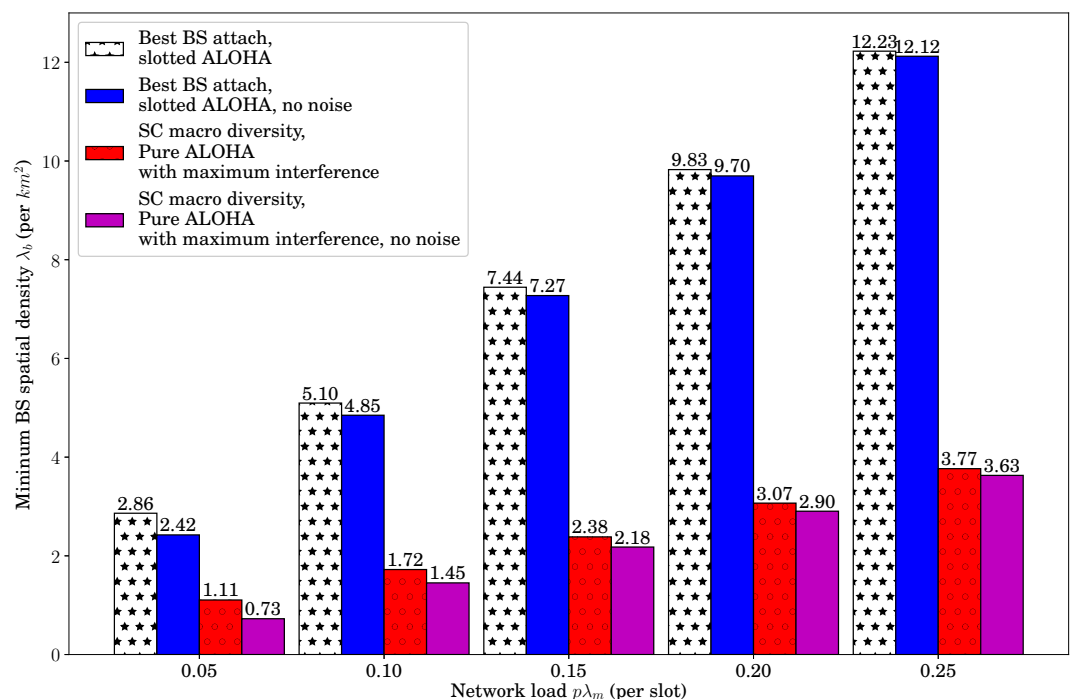

Fig. 2. Minimum BS spatial density with respect to network load. The target packet loss rate and outage probability threshold are both $10 \%$.

We consider a LPWAN network with target network packet loss rate $10 \%$. With a given outage probability requirement, we calculate the minimum required BS spatial density (per $\mathrm{km}^{2}$ ) with respect to the network load (per slot). The latter varies from 0.05 to 0.3 . The numerical results are shown in Fig. 2 (with outage probability 10\%) and Fig. 3 (with outage probability $1 \%$ ).

From (15), we know that, if the background noise is neglected, there exists a linear relationship between network load (i.e. $p \lambda_{m}$ ) and minimum BS spatial density (i.e. $\lambda_{b}$ ) if target network packet loss rate and outage probability is given. This can be confirmed in Fig. 2 and Fig. 3. For slotted ALOHA based 


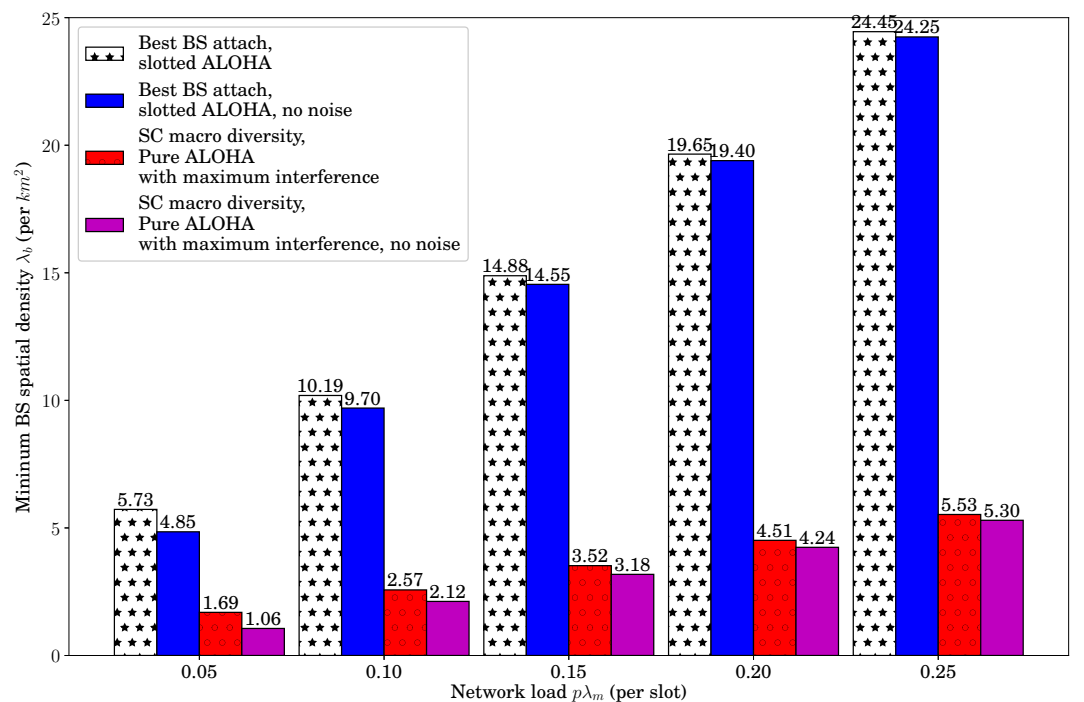

Fig. 3. Minimum BS spatial density with respect to network load. The target packet loss rate is $10 \%$ and outage probability threshold is $1 \%$.

best Best BS attach with background noise, we unfortunately have no closedform expression between minimum required BS density and network load. From these two figures, the relationship between minimum required BS density and network load can be assumed to be linear and we can use curve fitting techniques to get a closed form expression for the case with noise with high accuracy. In addition, It is not surprised that with background noise, the minimum required BS density is more larger.

From these two figures, we observe that as network load increases, the increase of minimum BS spatial density in case of macro diversity is significantly smaller than that in case of best BS attach. One straightforward explanation is: to guarantee a certain level of outage probability, as the load increases, the network should be more dense and the distance between a considered device and its surrounding BS is closer. The chance that one packet is received by several surrounding BS is thus much higher in a dense network. Thus, macro diversity has more obvious effect.

\section{Conclusion}

In this paper, we study the performance of selection combining macro diversity in LPWAN with outage probability constraint. The outage probability is a performance indicator that better takes into account the real availability of the service in any part of the area. We compare the minimum required BS spatial density between best BS attach and SC macro diversity to confirm the interest of applying selection combining macro diversity. From numerical result, we observe that minimum required BS density and network load can be assumed to 
be linear if best BS attach method is applied and outage probability constraint is given. SC macro diversity is more resistant to such a constraint.

\section{References}

1. Andrews, J.G., Baccelli, F., Ganti, R.K.: A tractable approach to coverage and rate in cellular networks. IEEE Transactions on Communications 59(11), 31223134 (2011)

2. Baccelli, F., Blaszczyszyn, B., Muhlethaler, P.: An Aloha protocol for multihop mobile wireless networks. IEEE Transactions on Information Theory 52(2), 421436 (2006)

3. Błaszczyszyn, B., Mühlethaler, P.: Stochastic analysis of non-slotted ALOHA in wireless ad-hoc networks. In: INFOCOM, 2010 Proceedings IEEE. pp. 1-9. IEEE (2010)

4. Dhillon, H.S., Andrews, J.G.: Downlink rate distribution in heterogeneous cellular networks under generalized cell selection. IEEE Wireless Communications Letters 3(1), 42-45 (2014)

5. Georgiou, O., Raza, U.: Low power wide area network analysis: Can LoRa scale? IEEE Wireless Communications Letters 6(2), 162-165 (2017)

6. Haenggi, M., Ganti, R.K.: Interference in large wireless networks. Now Publishers Inc (2009)

7. Jung, P., Steiner, B., Stilling, B.: Exploitation of intracell macrodiversity in mobile radio systems by deployment of remote antennas. In: Spread Spectrum Techniques and Applications Proceedings, 1996., IEEE 4th International Symposium on. vol. 1, pp. 302-307. IEEE (1996)

8. Lagrange, X., Godelewski, P., Tabbane, S.: Réseaux GSM: des principesa la norme (2000)

9. Pena-Martin, J.P., Romero-Jerez, J.M., Tellez-Labao, C.: Performance of Selection Combining Diversity in $\eta-\mu$ Fading Channels With Integer Values of $\mu$. IEEE Transactions on Vehicular Technology 64(2), 834-839 (2015)

10. Raza, U., Kulkarni, P., Sooriyabandara, M.: Low power wide area networks: An overview. IEEE Communications Surveys \& Tutorials 19(2), 855-873 (2017)

11. Song, Q., Lagrange, X., Nuaymi, L.: Evaluation of Macro Diversity Gain in Long Range ALOHA Networks. IEEE Communications Letters (2017)

12. Song, Q., Nuaymi, L., Lagrange, X.: Survey of radio resource management issues and proposals for energy-efficient cellular networks that will cover billions of machines. EURASIP Journal on Wireless Communications and Networking 2016(1), $1-20(2016)$

13. Sornin, N., Luis, M., Eirich, T., Kramp, T.: LoRaWAN specification. Tech. Rep. V.1.0, LoRa Alliance (Jan 2015), https://www.lora-alliance.org/portals/0/ specs/LoRaWAN\%20Specification\%201R0.pdf .

14. Vaze, R.: Random Wireless Networks: An Information Theoretic Perspective. Cambridge University Press (2015), https://books.google.fr/books?id= PSfZrQEACAAJ 